\title{
Multisession CyberKnife Radiosurgery for Symptomatic Abducens Nerve Palsy
}

\author{
Yuko Harada*1 and Shinichiro Miyazaki ${ }^{2}$ \\ ${ }^{1}$ Division Head of General Internal Medicine, Yamato Tokushukai Hospital, Japan \\ ${ }^{2}$ Director of Radiation Oncology, Shin-yurigaoka General Hospital, Japan \\ *Corresponding author: Yuko Harada, Division Head of General Internal Medicine, Yamato Tokushukai Hospital, Japan
}

\begin{tabular}{|c|c|}
\hline ARTICLE INFO & ABSTRACT \\
\hline & \multirow{3}{*}{$\begin{array}{l}\text { Abducens nerve palsy causes diplopia which lowers patients' quality of life. Nerve } \\
\text { palsy due to tumor compression is treated by surgery, but Abducens nerve is difficult } \\
\text { to access. Ten patients with benign and malignant tumors in cavernous sinus and cliva } \\
\text { were involved in this study: trigeminal schwannoma, meningioma, cavernous hemangio- } \\
\text { ma, metastatic tumors from prostate, breast, and uterus cancer. They were treated with } \\
\text { multisession CyberKnife radiosurgery on outpatient bases. Diplopia was treated in all } \\
\text { the patients, without any significant adverse effects. The longest follow-up was over } 10 \\
\text { years, with all patients leading normal lives. This is the first study to evaluate the use of } \\
\text { CyberKnife radiosurgery for the treatment of diplopia. }\end{array}$} \\
\hline Published: 慧 April 02, 2019 & \\
\hline $\begin{array}{l}\text { Citation: Yuko Harada, Shinichiro Mi- } \\
\text { yazaki. Multisession CyberKnife Ra- } \\
\text { diosurgery for Symptomatic Abducens } \\
\text { Nerve Palsy. Biomed J Sci \& Tech Res } \\
\text { 16(4)-2019. BJSTR. MS.ID.002895. }\end{array}$ & \\
\hline & $\begin{array}{l}\text { Abbreviations: QOI: Quality Of Life; SBRT: Stereotactic Body Radiotherapy; GTV: Gross } \\
\text { Tumor Volume; CTV: Clinical Target Volume; PTV: Planning Target Volume; ADL: Activi- } \\
\text { ties of Daily Living }\end{array}$ \\
\hline
\end{tabular}

\section{Introduction}

There are many causes for Abducens nerve palsy. Diplopia due to Abducens Nerve palsy not only causes mental distress to the patients but also lowers quality of life (QOL) in disablement of reading and writing.

Nerve palsy due to compression by tumor can be treated by removing or downsizing the tumor, usually with surgery or radiation therapy. Abducens nerve is in the deep part of the brain which is difficult to reach. Standard surgery is either impossible or harmful, while standard radiation therapy takes several weeks of treatment. The CyberKnife system (Accuracy Incorporated, Sunnyvale, California) is a robotic radiosurgery system that provides highly precise stereotactic body radiotherapy (SBRT). SBRT with CyberKnife is far less invasive compared to standard surgery, can be completed within 1 week, and hospitalization is not required. Abducens nerve palsy due to tumor is shown to be sufficiently treated by CyberKnife.

\section{Materials and Methods}

Ten cases were treated with CyberKnife since 2006 as shown in Table 1. Seven cases had benign tumors (Trigeminal schwannoma, meningioma, and cavernous hemangioma) and 3 cases had metastatic cancer (breast cancer, prostate cancer, and uterus cancer) which caused Abducens nerve palsy. Standard surgery was difficult in all cases, as the tumors were difficult to reach located in the cliva or cavernous sinus, thus the patients continued to suffer from diplopia.

All the patients had contrast-enhanced brain MRI and contrast-enhanced brain CT scans to evaluate the entire brain prior to the treatment. Tumors were tracked with skull tracking algorithm. The gross tumor volume (GTV) was defined as visible tumor on enhanced MRI with images merged for target definition. GTV was considered the same as clinical target volume (CTV). The planning target volume (PTV) included CTV and a margin of $1.2 \mathrm{~mm}$. 
Table 1: 10 cases treated with CyberKnife. TS: Trigeminal schwannoma. CH: cavernous hemangioma. Ca: cancer. CS: cavernous sinus. PR: partial remission. CR: complete remission.

\begin{tabular}{|c|c|c|c|c|c|c|c|c|c|c|}
\hline Case & $\mathbf{1}$ & $\mathbf{2}$ & $\mathbf{3}$ & $\mathbf{4}$ & $\mathbf{5}$ & $\mathbf{6}$ & $\mathbf{7}$ & $\mathbf{8}$ & $\mathbf{9}$ & $\mathbf{1 0}$ \\
\hline Histology & TS & TS & Meningioma & CH & TS & Neningioma & Meningioma & Breast Ca & Prostate Ca & Uterus Ca \\
\hline Location & CS & CS & CS & CS & CS & Cliva & Cliva & Cliva & Cliva & Cliva \\
\hline Age & 57 & 41 & 51 & 32 & 40 & 76 & 54 & 45 & 59 & 67 \\
\hline Sex & M & M & F & F & F & M & M & F & M & F \\
\hline PTV (cc) & 10.8 & 6.7 & 5.4 & 19.8 & 6.3 & 18.6 & 20.7 & 9.1 & 4.8 & 4.8 \\
\hline Dose (Gy) & 20 & 20 & 24.5 & 25 & 27 & 25.6 & 25 & 35 & 40 & 38 \\
\hline Fraction & 3 & 3 & 5 & 5 & 5 & 8 & 5 & 8 & 5 & 5 \\
\hline Outcome & PR & PR & PR & PR & PR & PR & PR & CR & CR & CR \\
\hline
\end{tabular}

The treatments were on outpatient bases, and followed up every 4 to 6 months with MRI or PET/CT scan.

\section{Results/Observations}

PTV ranged from $4.8 \mathrm{cc}$ to $20.7 \mathrm{cc}$ (average $10.7 \mathrm{cc}$ ), and radiation dose ranged from 20 Gy to $40 \mathrm{~Gy}$ (average $28 \mathrm{~Gy}$ ). Fraction ranged from 3 to 8 (average 5.2).

All cases showed CR or PR, thus demonstrated improvement in diplopia. No adverse event over Grade 3 was observed.

A 76-year-old male was diagnosed with large meningioma in cliva (Figure 1). He developed gait disturbance, diplopia (Abducens

nerve palsy), and dysphagia. Since he had cardiac dysfunction, the surgery was difficult. He underwent CyberKnife radiosurgery with 25.6 Gy in 8 fractions. The tumor decreased in volume over 1 year, and the symptoms disappeared as the tumor became smaller. At 3 years follow-up, the tumor was stable and had not grown back.

A 32-year-old female was diagnosed with cavernous haemangioma (Figure 2). Since standard surgery was impossible for her, she thus continued to suffer from diplopia due to Abducens nerve palsy. However, she was able to undergo CyberKnife radiosurgery with 25 Gy in 5 fractions. At 18-months follow-up, the tumor decreased in size and diplopia disappeared.

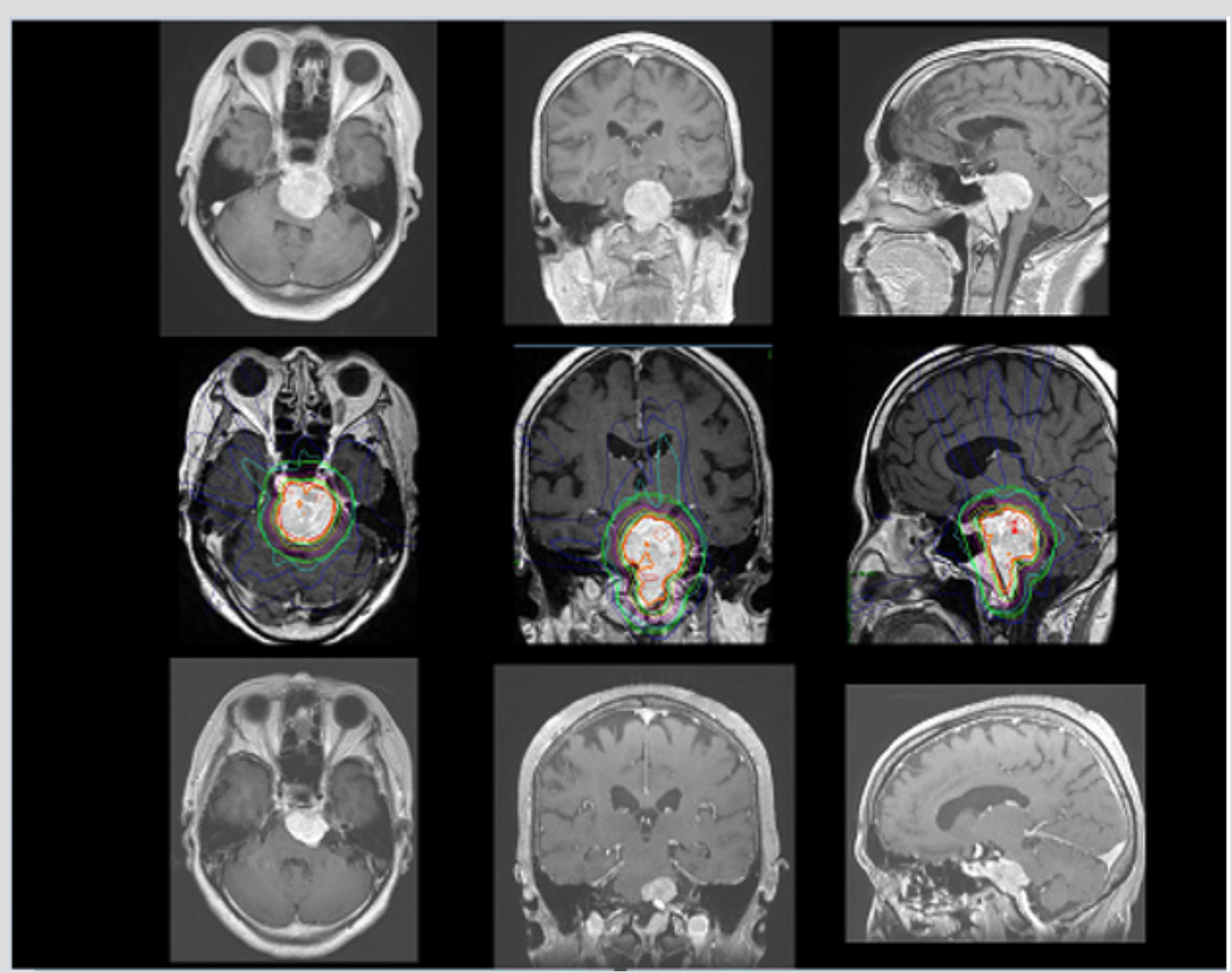

Figure 1: A 76 years old male with meningioma in cliva (Case 6 in Table 1). Top: brain MRI before treatment. Middle: treatment plan for meningioma. Bottom: 36 months after treatment. The tumor decreased in size. 


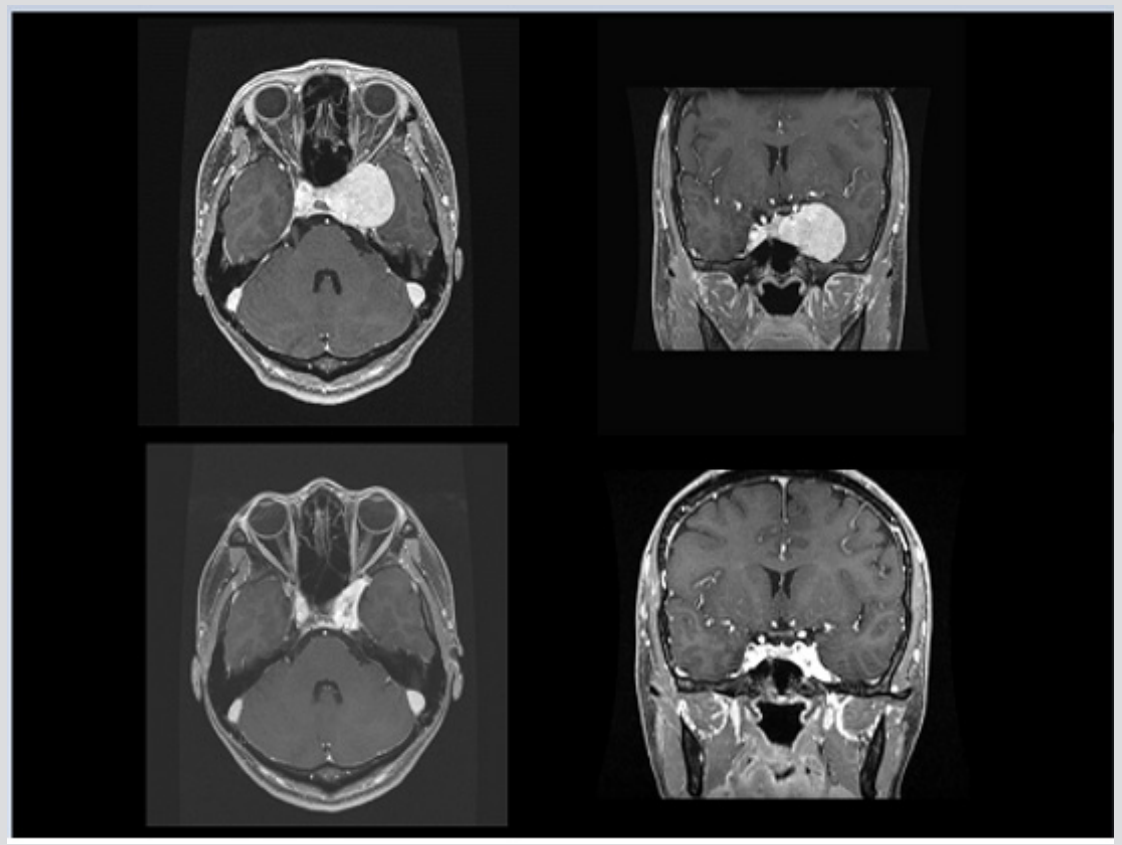

Figure 2: A 32 years old female with cavernous hemangioma (Case 4 in Table 1). Top: brain MRI before treatment. Bottom: 18 months after treatment. The tumor remarkably decreased in size.

\section{Discussion}

All the benign tumors had PR, whereas all the cancers had CR. One of the reasons for only PR of the benign tumors was that they were much larger than cancers in this study. Another is that some of these benign tumors are less susceptible to radiation. Regardless of PR or CR, all the patients had improvement in double vision with no relapse. The aim of this study was to investigate whether CyberKnife is beneficial to treat diplopia caused by tumor compression, and we had excellent results. Then, is CyberKnife treatment appropriate for benign tumor?

Schwannomas are benign tumors originating from the Schwann cells of the nerve sheaths. Trigeminal schwannomas are rare tumors, accounting for $1-2 \%$ of all intracranial schwannomas and tend to occur predominantly in the 4th-6th decades [1]. The lesions are solitary, slow growing, and generally not associated with neurologic symptoms [2]. The standard treatment is surgery, and these tumors were said to be radioresistant [2,3]. However, Kharod et al. reported recently that fractionated radiation therapy for benign nonacoustic schwannoma to a total dose of 50 Gy provided excellent local control and minimal morbidity [4]. Our 3 cases of trigeminal schwannomas were all in the 40s and 50s, suffering from diplopia due to this slow-progressing tumor. If well-designed, radiation therapy can be effective, adequate, and far less-invasive treatment for trigeminal schwannoma.

We had 3 cases of meningiomas, 2 in cavernous sinus and 1 in cliva. Meningiomas are the most common type of extraaxial primary brain tumor, representing a third of all primary central nervous system neoplasms [5]. Primary treatment is total resection, but cavernous sinus and cliva are very difficult to approach. Day SE et al. reported in their review that surgical resection of cavernous sinus meningioma was often associated with cranial neuropathies, and radiotherapy alone for grade I meningioma showed excellent local control rates ranging from $89-99 \%$ at 5 years and $79-97 \%$ at 10 years [6]. These strongly support CyberKnife treatment for meningioma in cavernous sinus and cliva.

We also had 1 case of cavernous hemangioma. Wang X et al. reported that multisession CyberKnife radiosurgery (1-4 fractions) for 45 patients with cavernous sinus hemangiomas showed excellent medium and long-term local tumor control with minimal adverse effects [7]. Their study is the only one in the previous publishment using CyberKnife for cavernous sinus hemangioma. During their follow-up for 22-70 months, 15 patients showed more than $80 \%$ decrease in tumor size, 18 patients showed $60-80 \%$ decrease, and 12 patients showed $40-60 \%$ decrease. They were not able to eradicate the tumors, but the tumor control was satisfactory for such benign tumors.

The rest of the 3 cases had metastatic tumor of breast, prostate, and uterus cancer. All of them underwent surgical treatment and chemotherapy for original cancer, however their cancers metastasized in the brain thereby causing diplopia. After CyberKnife treatment, their diplopia was significantly improved.

This is a unique approach of SBRT using CyberKnife to treat diplopia which weakens patients' Activities of Daily Living (ADL) and decreases QOL. Whether it is caused by benign tumor or metastatic cancer, Abducens nerve palsy is tormenting and annoying for patients who want to live normal lives. 


\section{Conclusion}

Symptomatic Abducens nerve palsy due to compression of tumors can be treated with multisession CyberKnife radiosurgery even for those patients who are not indicated to standard surgery. The treatment can be done safely on outpatient basis, and significantly improves patients' ADL and QOL.

\section{References}

1. Agarwal A (2015) Intracranial Trigeminal schwannoma. Neuroradiol J 28(1): 36-41

2. Agrawal A, Singh V, Rohilla S, Sharma B (2017) Trigeminal schwannoma. Natl J Maxillofac Surg 8(2): 149-152.

ISSN: 2574-1241

DOI: 10.26717/BJSTR.2019.16.002895

Yuko Harada. Biomed J Sci \& Tech Res

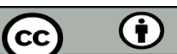

This work is licensed under Creative Commons Attribution 4.0 License

Submission Link: https://biomedres.us/submit-manuscript.php
3. Artzi Z, Taicher S, Nass D (1991) Neurilemmoma of the mental nerve. J Oral Maxillofac Surg 49: 196-200.

4. Kharod SM, Herman MP, Amdur RJ, Mendenhall WM (2018) Fractionated radiation therapy for benign nonacoustic schwannomas. Am J Clin Oncol 4(1): 13-17.

5. Whittle IR, Smith C, Navoo P (2004) Meningiomas. Lancet 363: 15351543.

6. Day SE, Halasz LM (2017) Radiation therapy for WHO grade I meningioma. Chin Clin Oncol 6(1): S4.

7. Wang X Wang E, Liu X, Pan L, Dai J, et al. (2015) Multisession cyberknife radiosurgery for cavernous sinus hemangiomas: medium- and longterm outcomes. Zhonghua Wai Ke Za Zhi 53(10): 767-771.

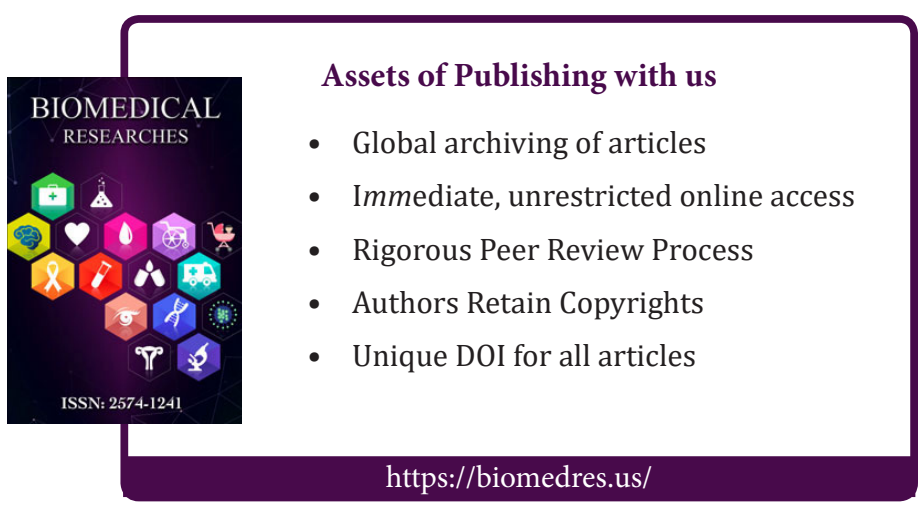

